\title{
Cooling to the Ground State of Axial Motion for One Atom Strongly Coupled to an Optical Cavity
}

\author{
A. D. Boozer, A. Boca, R. Miller, T. E. Northup, and H. J. Kimble \\ Norman Bridge Laboratory of Physics 12-33, California Institute of Technology, Pasadena, California 91125, USA
}

(Received 11 June 2006; published 23 August 2006)

\begin{abstract}
Localization to the ground state of axial motion is demonstrated for a single, trapped atom strongly coupled to the field of a high finesse optical resonator. The axial atomic motion is cooled by way of coherent Raman transitions on the red vibrational sideband. An efficient state detection scheme enabled by strong coupling in cavity QED is used to record the Raman spectrum, from which the state of atomic motion is inferred. We find that the lowest vibrational level of the axial potential with zero-point energy $\hbar \omega_{a} / 2 k_{B}=13 \mu \mathrm{K}$ is occupied with probability $P_{0} \simeq 0.95$.
\end{abstract}

DOI: $10.1103 /$ PhysRevLett.97.083602

PACS numbers: 42.50.Pq, 03.67.- a, 32.80.Pj

Single atoms strongly coupled to the fields of high quality optical resonators are of fundamental importance in quantum optics and, more generally, can be used for many tasks in quantum information science, including the implementation of scalable quantum computation [1,2] and the realization of distributed quantum networks $[3,4]$. In recent years, significant experimental progress to develop tools suitable for these tasks has been made by employing optical forces to localize individual atoms within optical cavities in a regime of strong coupling [511], as well as by combining trapped ions with optical cavities [12]. Scientific advances thereby enabled include the observation of the vacuum-Rabi spectrum for an individual atom [9] and vacuum-stimulated cooling [10].

Although great strides are being made with atoms localized and strongly coupled to the fields of optical cavities, it has not previously been possible to access the quantum regime for the atomic center-of-mass motion in cavity QED. Qualitatively new phenomena have been predicted in this regime for which a quantized treatment is required for both the internal (i.e., the atomic dipole and cavity field) and external (i.e., atomic motion) degrees of freedom, as was first recognized in the seminal work of Refs. [13-15] and in the years since [16-22]. Examples include the transfer of quantized states of atomic motion to quantum states of light, and conversely [22], as well as for measurements that surpass the standard quantum limit for sensing atomic position [16].

Our effort towards quantum control of atomic motion in cavity QED follows the remarkable set of achievements for trapped ions [23] and atoms in optical lattices [24], for which such control has led to the creation of manifestly quantum states of motion and to the manipulation of quantum information. A first step in many of these investigations has been the capability to cool to the ground state of motion for single trapped atoms or ions.

In this Letter, we report localization to the ground state of motion for one atom trapped in an optical cavity in a regime of strong coupling [11]. Resolved sideband cooling to the ground state is accomplished with a coherent pair of intracavity Raman fields. To deduce the resulting state of atomic motion, we introduce a scheme for recording Raman spectra by way of the interaction of the atom with a resonant cavity probe. Our scheme is the cavity QED equivalent of state detection in free space by quantum-jump spectroscopy [23] and achieves a confidence level for state discrimination $>98 \%$ in $100 \mu \mathrm{s}$. From the Raman spectra, we infer that the lowest vibrational level $n=0$ of the axial potential is occupied with probability $P_{0} \simeq 0.95$ for one trapped atom.

A schematic of the experiment is given in Fig. 1. At the heart of the system is the Fabry-Perot cavity formed by mirrors $\left(M_{1}, M_{2}\right)$. The cavity length is stabilized to $l_{0}=$ $42.2 \mu \mathrm{m}$ using an independent locking laser, such that a $\mathrm{TEM}_{00}$ mode is resonant with the $6 S_{1 / 2}, F=4 \rightarrow 6 P_{3 / 2}$, $F^{\prime}=5$ transition of the $D 2$ line in Cs. The resulting atomcavity coupling gives a maximum single-photon Rabi frequency of $2 g_{0} / 2 \pi=68 \mathrm{MHz}$ for $\left(F=4, m_{F}= \pm 4\right) \rightarrow$ $\left(F^{\prime}=5, m_{F}^{\prime}= \pm 5\right)$. The decay rates are $\gamma / 2 \pi=2.6 \mathrm{MHz}$ for the $6 P_{3 / 2}$ excited states and $\kappa / 2 \pi=4.1 \mathrm{MHz}$ for the cavity field. Because $g_{0} \gg(\gamma, \kappa)$, our system is in the strong coupling regime of cavity QED [11], with critical

(a)

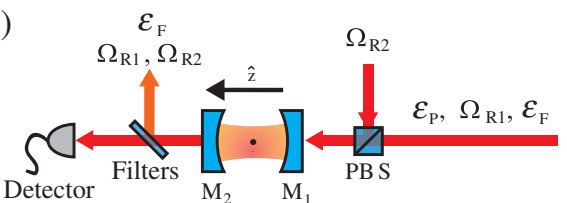

(b)

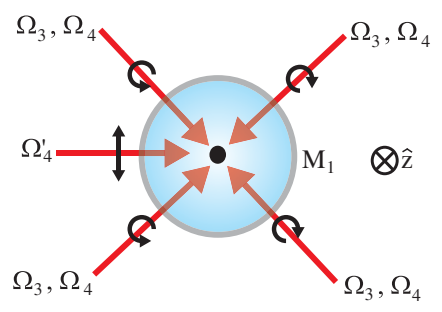

FIG. 1 (color online). Schematic of the experiment. The cavity is represented (a) from the side and (b) along its axis. Shown are the various beams used in the experiment: linearly polarized probe $\mathcal{E}_{p}$, FORT $\mathcal{E}_{F}$, pumping $\Omega_{4}^{\prime}$, and Raman beams $\Omega_{R 1, R 2}$, as well as the circularly polarized lattice beams $\Omega_{3,4}$. 
photon and atom numbers $n_{0} \equiv \gamma^{2} /\left(2 g_{0}^{2}\right) \approx 0.0029$ and $N_{0} \equiv 2 \kappa \gamma / g_{0}^{2} \approx 0.018$.

Atoms are trapped by an intracavity far-off-resonant trap (FORT) at $\lambda_{F}=935.6 \mathrm{~nm}$, which is driven by a linearly polarized input field $\mathcal{E}_{F}$ and is resonant with a $\mathrm{TEM}_{00}$ mode of the cavity with linewidth $\kappa_{F} / 2 \pi=0.8 \mathrm{GHz}$. At $\lambda_{F}$, states in the ground $F=3,4$ and excited state $F^{\prime}=5$ manifolds experience nearly equal trapping potentials. For states in the $F=3$, 4 manifolds, this potential is independent of $m_{F}$ and has a peak value of $U_{F} / h=-41 \mathrm{MHz}$, while for states in the $F^{\prime}=5$ manifold it has a weak dependence on $m_{F}^{\prime}[6,9]$. The standing-wave structure of the FORT forms independent wells where atoms may be trapped. Near the bottom of a FORT well, the potential is approximately harmonic, with axial and radial vibrational frequencies $\omega_{a} / 2 \pi=530 \mathrm{kHz}$ and $\omega_{r} / 2 \pi=4.5 \mathrm{kHz}$, respectively.

To load atoms into the FORT, we release a cloud of cold atoms located $\sim 3 \mathrm{~mm}$ above the cavity [6]. As the atoms fall through the cavity, we apply $5 \mathrm{~ms}$ of $\Omega_{3}, \Omega_{4}$ light by way of two pairs of counterpropagating $\sigma_{+}-\sigma_{-}$polarized beams; see Fig. 1(b). These beams are blue detuned by $+10 \mathrm{MHz}$ from the $F=3 \rightarrow F^{\prime}=3$ and $F=4 \rightarrow F^{\prime}=$ 4 transitions, respectively, and cool the falling atoms via polarization gradient cooling [25]. We adjust the powers [26] of these beams so that the probability of loading at least one atom is $\sim 0.3$ per cloud drop.

Raman coupling between the $F=3$ and $F=4$ manifolds is generated by driving a cavity mode at $\lambda_{R}=$ $945.6 \mathrm{~nm}$ with a pair of beams $\Omega_{R 1, R 2}$ that are phaselocked, lin $\perp$ lin polarized, and have a relative detuning $\omega_{R 1}-\omega_{R 2}=\Delta_{H F}^{\prime}+\delta_{R}$, where $\Delta_{H F}^{\prime} / 2 \pi=9.19261 \mathrm{GHz}$ is the Cs hyperfine splitting [27] and $\delta_{R}$ is the Raman detuning. This cavity mode has a linewidth $\kappa_{R} / 2 \pi=6 \mathrm{GHz}$, and the Raman beams are tuned such that $\Omega_{R 1(R 2)}$ lies $\Delta_{H F}^{\prime} / 2$ above (below) cavity resonance. Since $\Omega_{R 1, R 2}$ drive a different mode of the cavity than $\mathcal{E}_{F}$, atoms trapped in different FORT wells see different Raman powers. If the relative spatial phase along the cavity axis between the FORT and the Raman pair at the bottom of a given well is $\alpha$, then an atom at this potential minimum sees a Raman power proportional to $\cos ^{2} \alpha$.

We set the optical power transmitted on resonance through the cavity for $\Omega_{R 1, R 2}$ to $P_{R 1}=P_{R 2}=140 \mu \mathrm{W}$, which gives a Rabi frequency [28] $\Omega_{0} / 2 \pi=200 \mathrm{kHz}$ for atoms with $\alpha=0$. The ac-Stark shift due to these beams adds a correction to the FORT potential of $U_{R} / 2 \pi=$ $0.84 \mathrm{MHz}$. To avoid heating the atom by switching $U_{R}$, we leave $\Omega_{R 1, R 2}$ on all the time, but usually keep them far detuned $\left(\delta_{R} / 2 \pi=85 \mathrm{MHz}\right)$ from Raman resonance. To drive Raman transitions, we change $\omega_{R 2}$ to bring the pair into Raman resonance, whereas to fine-tune $\delta_{R}$ we vary $\omega_{R 1}$.

Because the intensity of the Raman pair is spatially varying, the Raman coupling can connect states with different vibrational quantum numbers. The form of this mo- tional coupling is simple for atoms near the bottom of a FORT well where the axial and radial motions decouple, allowing us to consider the effect of the Raman coupling on the axial motion alone. In this harmonic limit, we can define a set of Fock states $\{|n\rangle\}$ for the axial motion. For transitions from $F=3, m=0$ to $F=4, m=0$ and to first order in $\eta$, the Rabi frequency for an $n \rightarrow n$ transition is $\Omega_{n \rightarrow n}=(1 / 2)(1+\cos 2 \alpha) \Omega_{0}$, while for an $n \rightarrow n-1$ transition, $\Omega_{n \rightarrow n-1}=\eta \sqrt{n} \sin 2 \alpha \Omega_{0}$, where $\eta=\left(2 \pi / \lambda_{R}\right) \sqrt{\hbar / 2 m \omega_{a}}=0.056$ is the Lamb-Dicke parameter for axial motion. Note that the $n \rightarrow n-1$ transition is strongest for atoms with $\alpha=\pi / 4$.

The spatial dependence of the Raman coupling, together with the fact that the axial motion of the atom is in the Lamb-Dicke limit, allows us to implement Ramansideband cooling [23]. We tune the Raman pair to the red axial sideband $\left(\delta_{R}=-525 \mathrm{kHz} \simeq-\omega_{a}\right)$ and apply the $\Omega_{4}$ lattice beams. An atom that starts in $F=3$ is coherently transferred by $\Omega_{R 1, R 2}$ to $F=4$, where it is incoherently repumped to $F=3$ by $\Omega_{4}$. The coherent transfer lowers the axial vibrational quantum number $n$ by one, while the incoherent repumping usually leaves $n$ unchanged, since $n$-changing transitions are Lamb-Dicke suppressed. Thus, the beams continually lower $n$, cooling the atom to the axial ground state. Also, the $\Omega_{4}$ light provides Sisyphus cooling [25] in the radial direction.

Strong atom-cavity coupling enables a versatile detection scheme for determining if an atom is present in the cavity and, if so, if it is in the $F=3$ or $F=4$ manifold. In $100 \mu \mathrm{s}$, we measure the atomic hyperfine state with a confidence level of $>98 \%$. The scheme involves driving the cavity with a $100 \mu$ s pulse of resonant $F=4 \rightarrow F^{\prime}=$ 5 probe light $\mathcal{E}_{P}$. If an $F=3$ atom is present (or if the cavity is empty), then the light is transmitted, while if an $F=4$ atom is present, then the light is blocked because of strong coupling [9]. We set the $\mathcal{E}_{P}$ intensity such that on average $N_{e}$ photons are detected [29] per probing interval with no atom in the cavity. As shown in Fig. 2, if the number $N$ of detected photons is such that $N<0.25 N_{e}$,

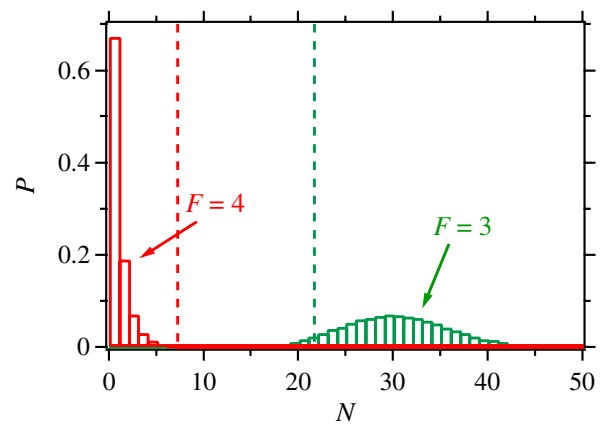

FIG. 2 (color online). Histogram of counts recorded during $100 \mu$ s probing intervals for $N_{e}=30$. The left (right) curve is the probability $P$ of detecting a number of counts $N$ for an atom prepared in the $F=4(F=3)$ state. The dashed lines indicate detection thresholds at $N=0.25 N_{e}$ and $N=0.75 N_{e}$. 
we assume an $F=4$ atom is present; if $N>0.75 N_{e}$, we assume an $F=4$ atom is not present; otherwise, the measurement is inconclusive $(<2 \%$ of the time) and we ignore the result. Whenever we detect the atomic state, we perform two such measurements: the first with $\mathcal{E}_{P}$ to find out if an $F=4$ atom is present, the second with $\mathcal{E}_{P}$ together with $\Omega_{3}$ as a repumper [26], to detect an atom, regardless of its internal state.

We measure the Raman transfer probability $P_{4}$ for a given $\delta_{R}$ by preparing an atom in $F=3$, applying a Raman pulse, and then detecting the atomic state using the above scheme (with $N_{e} \sim 22$ ). For each measurement cycle (or trial), we first Raman-sideband cool the atom for an interval $\Delta t_{c}$. Next, we pump it into $F=3$ by alternating $1 \mu \mathrm{s}$ pulses of $\Omega_{4}$ lattice light with $1 \mu \mathrm{s}$ pulses of $\Omega_{4}^{\prime}$ linearly polarized resonant $F=4 \rightarrow F^{\prime}=4$ light from the side of the cavity (10 pulses of each). After the atom is pumped to $F=3$, we apply a $\Delta t_{R}=500 \mu$ s Raman pulse, which sometimes transfers it to $F=4$. Finally, we measure the atomic state and check if the atom is still present. For each atom, we fix the absolute value of the Raman detuning $\left|\delta_{R}\right|$ and alternate trials at $+\left|\delta_{R}\right|$ with trials at $-\left|\delta_{R}\right|$ (299 trials each). By combining data from atoms with different values of $\left|\delta_{R}\right|$, we map out a Raman spectrum. Note that, because the initial Zeeman state of the atom is random, all allowed $F=3 \rightarrow F=4$ Zeeman transitions contribute to these spectra.

Two example Raman spectra are plotted in Fig. 3. For the curve in Fig. 3(a), we cool for $\Delta t_{c}=250 \mu \mathrm{s}$, for the curve in Fig. 3(b) for $\Delta t_{c}=5 \mathrm{~ms}$. These scans are performed after nulling the magnetic field to within $\sim 40 \mathrm{mG}$; the widths of the peaks are set by the splitting of different Zeeman levels due to the residual magnetic field. For the curve in Fig. 3(a), we see peaks at the carrier $\left(\delta_{R}=0\right)$, as well as at the blue/red sidebands $\left(\delta_{R} / 2 \pi \simeq \pm 530 \mathrm{kHz}=\right.$ $\left.\pm \omega_{a}\right)$. Already we note a sideband asymmetry, indicating that a significant fraction of the population is in the $n=0$ vibrational state. For the data in Fig. 3(b), the red sideband at $\delta_{R} / 2 \pi \simeq-530 \mathrm{kHz}$ is suppressed such that it cannot be distinguished from the background and contribution from off-resonant excitation of the carrier.

The ratio $r$ of transfer probabilities for the red and blue sidebands gives information about the temperature of the atom. For a two-state atom in a thermal state, this ratio $r_{0}$ at $\left|\delta_{R}\right|=\omega_{a}$ is related to the mean vibrational quantum number $\bar{n}$ by $r_{0}=\bar{n} /(\bar{n}+1)$ [23]. In Fig. 3(c), we plot $r$ as a function of $\left|\delta_{R}\right|$ for the $\Delta t_{c}=5 \mathrm{~ms}$ data. As shown in Fig. 3(b), we fit a Lorentzian curve to the carrier, then subtract its contribution from both the red and the blue sideband data, as shown in Fig. 3(c). We find $r_{0} \simeq \bar{n}=$ $0.01 \pm 0.05$, and the ground state population $P_{0}=1 /(\bar{n}+$ 1) $=0.99 \pm 0.05$, where the error bars reflect fluctuations in the data around $\left|\delta_{R}\right|=\omega_{a}$. If, instead, we subtract the constant background of $P_{4}^{B}=0.024$ but not the carrier's Lorentzian tail, we find $r_{0} \simeq \bar{n}=0.05 \pm 0.04$ and $P_{0}=$ $0.95 \pm 0.04$. Finally, if we use the raw data from Fig. 3(b)
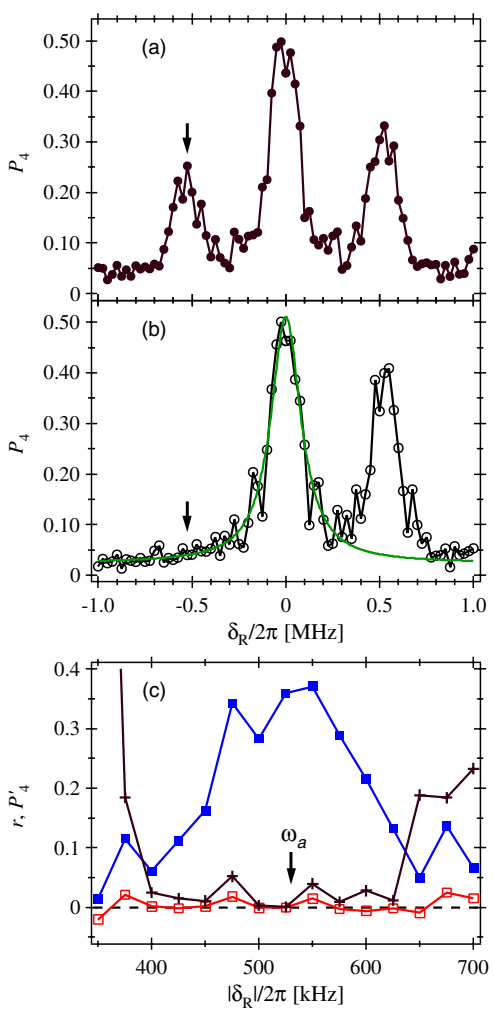

FIG. 3 (color online). Population $P_{4}$ in the $F=4$ state versus Raman detuning $\delta_{R} / 2 \pi$. The data in (a) are taken with $\Delta t_{c}=$ $250 \mu \mathrm{s}$ of cooling and an $\Omega_{4}$ total 4-beam intensity $I_{4}=5 I_{4}^{\text {sat }}$; those in (b) with $\Delta t_{c}=5 \mathrm{~ms}, I_{4}=0.5 I_{4}^{\text {sat }}$ (on average, $\sim 33$ atoms per data point). The arrow marks the detuning used for sideband cooling. (c) Zoom-in on the two sideband regions for the data in (b), with detuning axis folded around $\delta_{R}=0$. The red $(\square)$ and blue ( $\square$ ) sidebands, and their ratio $r$ $(+)$, are shown after subtracting a Lorentzian fit to the carrier [superimposed in (b)].

with no subtractions, we obtain $r_{0}=0.10 \pm 0.03, \bar{n}=$ $0.12 \pm 0.04$, and $P_{0}=0.89 \pm 0.03$. Because the atom is not a two-state system and the motional state is not known to be thermal, these estimates are approximate.

The axial cooling rate and asymptotic value of $\bar{n}$ depend on $\delta_{R}$, on the $\Omega_{R 1, R 2}$ Rabi frequencies, and on the power and detuning of $\Omega_{4}$. We have performed computer simulations to help us choose optimal values for these parameters. A common feature of both our theoretical and experimental investigations is the robustness of $\bar{n}$ under variations of the cooling parameters. As an example [30], in Fig. 4 we plot the measured sideband ratio $r_{0}$ at $\delta_{R} / 2 \pi=-500 \mathrm{kHz} \simeq-\omega_{a}$ as a function of (a) the detuning $\delta_{R}$ used for sideband cooling and (b) the recycling intensity $I_{4}$. The sideband asymmetry is maintained over a range of at least $200 \mathrm{kHz}$ in detuning and of 2 orders of magnitude in the intensity $I_{4}$ of the $\Omega_{4}$ beams. The insets give results from a 2-state calculation of $r_{0}$, displaying similar insensitivity to the exact values of $\delta_{R}$ and $I_{4}$.

We use two methods for estimating the mean energy $E_{r}$ for radial motion. The first method involves adiabatically lowering the FORT depth to zero, so that only the $U_{R}$ 

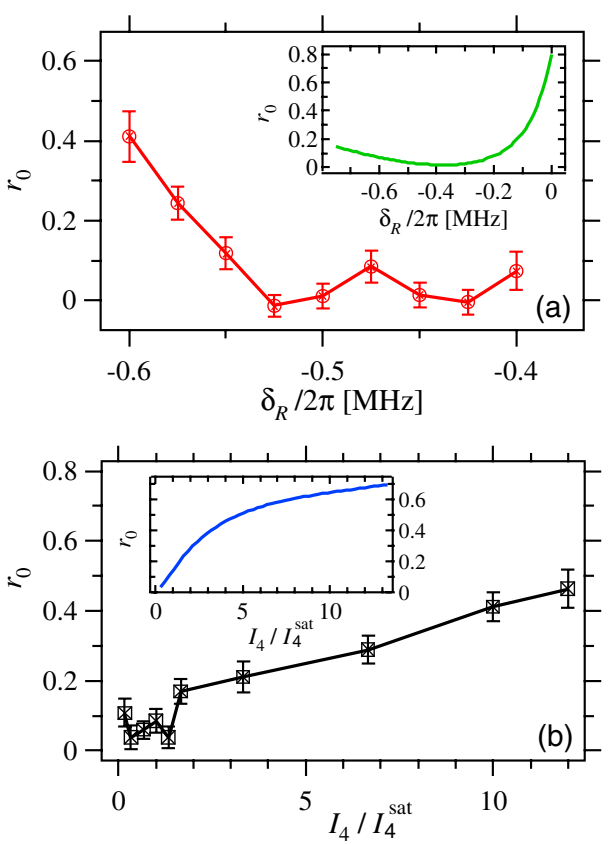

FIG. 4 (color online). Varying cooling parameters. The sideband ratio $r_{0}$ is shown as a function of (a) the Raman detuning $\delta_{R}$ employed for cooling and (b) the $4 \rightarrow 3$ repumping intensity $I_{4}$ [30]. Insets show the results from a simple calculation for a 2 state atom trapped in a FORT well with $\alpha=\pi / 4$.

trapping potential remains, and measuring the probability that the atom survives the process [31]. If we assume a thermal state for the radial motion, this method limits $E_{r} \lesssim$ $200 \mu \mathrm{K}$. The second method relies on the fact that radial motion would shift and/or broaden the axial sidebands in our Raman spectra. By applying a small axial bias field, we can resolve motional sidebands of transitions between specific Zeeman levels; the positions and widths of these sidebands also limit $E_{r} \lesssim 200 \mu \mathrm{K}$. However, the Sisyphus cooling we use radially has been previously shown to reach temperatures of $\sim 1 \mu \mathrm{K}$ [25], which corresponds to $n_{\text {rad }} \simeq$ 4 for an atom in our FORT. A possibility for improved determination of the radial "temperature" is to monitor the transmission of the cavity probe field, as in Ref. [9]. Note that the ratio of radial to axial trapping frequencies $(\simeq 0.01)$ is such that any modulation of the axial frequency due to radial motion would be adiabatic. As a result, we expect that an atom cooled to the axial ground state does not change its state due to radial motion.

In conclusion, we have demonstrated cooling to the ground state of axial motion for single cesium atoms strongly coupled to the field of a small optical resonator. Together with existing capabilities for strong coupling of the internal degrees of freedom, control over the external center-of-mass motion in cavity QED enables a new set of phenomena to be explored at the light-matter interface. For example, arbitrary states of atomic motion can be prepared from the ground state by coherent Raman transitions [23], then mapped to the electromagnetic field by way of the strong atom-field coupling [22]. Investigations of sensing atomic motion at the standard quantum limit and feedback control now become feasible [16,32].

This research is supported by the NSF and by the DTO of the DNI.

[1] T. Pellizzari et al., Phys. Rev. Lett. 75, 3788 (1995).

[2] L.-M. Duan et al., Phys. Rev. Lett. 92, 127902 (2004).

[3] J. I. Cirac et al., Phys. Rev. Lett. 78, 3221 (1997).

[4] H.-J. Briegel et al., in The Physics of Quantum Information, edited by D. Bouwmeester et al. (Springer, Berlin, 2000), p. 192.

[5] J. Ye et al., Phys. Rev. Lett. 83, 4987 (1999).

[6] J. McKeever et al., Phys. Rev. Lett. 90, 133602 (2003).

[7] P. Maunz et al., Nature (London) 428, 50 (2004).

[8] J. A. Sauer et al., Phys. Rev. A 69, 051804(R) (2004).

[9] A. Boca et al., Phys. Rev. Lett. 93, 233603 (2004).

[10] S. Nußmann et al., Nature Phys. 1, 122 (2005).

[11] R. Miller et al., J. Phys. B 38, S551 (2005).

[12] G. R. Guthöhrlein et al., Nature (London) 414, 49 (2001); A. B. Mundt et al., Phys. Rev. Lett. 89, 103001 (2002).

[13] B. G. Englert et al., Europhys. Lett. 14, 25 (1991).

[14] S. Haroche et al., Europhys. Lett. 14, 19 (1991).

[15] M. J. Holland et al., Phys. Rev. Lett. 67, 1716 (1991).

[16] P. Storey, M. Collett, and D. F. Walls, Phys. Rev. Lett. 68, 472 (1992); R. Quadt, M. Collett, and D. F. Walls, ibid. 74, 351 (1995).

[17] A. M. Herkommer et al., Phys. Rev. Lett. 69, 3298 (1992); Quantum Semiclass. Opt. 8, 189 (1996).

[18] W. Ren et al., Phys. Rev. A 51, 752 (1995).

[19] M. O. Scully et al., Phys. Rev. Lett. 76, 4144 (1996).

[20] D. W. Vernooy and H. J. Kimble, Phys. Rev. A 55, 1239 (1997); 56, 4287 (1997).

[21] A. C. Doherty et al., Phys. Rev. A 57, 4804 (1998).

[22] A. S. Parkins and H. J. Kimble, J. Opt. B 1, 496 (1999); in Frontiers of Laser Physics and Quantum Optics, edited by Z. Xu et al. (Springer, Berlin, 2000), p. 132; Phys. Rev. A 61, 052104 (2000).

[23] D. Leibfried et al., Rev. Mod. Phys. 75, 281 (2003).

[24] S. E. Hamann et al., Phys. Rev. Lett. 80, 4149 (1998); H. Perrin et al., Europhys. Lett. 42, 395 (1998); V. Vuletic et al., Phys. Rev. Lett. 81, 5768 (1998).

[25] D. Boiron et al., Phys. Rev. A 53, R3734 (1996).

[26] For loading, the total power in the four lattice beams is $50 I_{4}^{\text {sat }}$ for $\Omega_{3}$ and $\Omega_{4}$, where $I_{4}^{\text {sat }} \sim 3.8 \mathrm{~mW} / \mathrm{cm}^{2}$. For detection, the $\Omega_{3}$ intensity is $5 I_{4}^{\text {sat }}$.

[27] $\Delta_{H F}^{\prime}$ for a trapped atom is slightly reduced compared to its free space value $\Delta_{H F}$, because the FORT potential for the $F=3$ manifold is slightly weaker than for $F=4$. For our FORT, the correction is $\Delta_{H F}^{\prime}-\Delta_{H F}=-21 \mathrm{kHz}$.

[28] Here $\Omega_{0}$ is the Rabi frequency for the transition $F=3$, $m=0, n=0 \rightarrow F=4, m=0, n=0$ where the quantization axis is along the cavity axis.

[29] The probability for a photon starting out within the cavity to be recorded by our detectors is $e \sim 0.06$.

[30] Unless otherwise noted, the settings for this measurement are $I_{4}=0.3 I_{4}^{\text {sat }}, \Delta t_{c}=5 \mathrm{~ms}, \delta_{R}=-500 \mathrm{kHz}, \Delta t_{R}=$ $50 \mu \mathrm{s} ; P_{4}^{B}=0.065$ was subtracted before computing $r_{0}$.

[31] W. Alt et al., Phys. Rev. A 67, 033403 (2003).

[32] A. Doherty and K. Jacobs, Phys. Rev. A 60, 2700 (1999). 